menure compost yielded $1.9 \mathrm{lb}$. per sq. ft. against $1.2 \mathrm{lb}$. for the best of a number of 'synthetic' composts. Other features of practical culture relate to the successful growing of tomatoes in cardboard boxes treated with 'Cuprinol', the lack of disparity in yield when top-dressings are given in solid or liquid form, and the use of sulphate of ammonia as the sole source of nitrogen for the tomato crop.

The severity of disease caused by the fungus Didymella lycopersici seems to have no relation to manurial treatment, and excesses of magnesium, boron and manganese did not appear to influence infection (P. H. Williams). A fascinating possibility of 'curing' plants of virus diseases has been investigated by $R$. Howles. A spray of I per cent tannic acid on two consecutive days reduced by 50 per cent the number of plants infected. Solanums infected with mosaic were 'cured' by exposure to a temperature of $105^{\circ} \mathrm{F}$. for twelve hours per day for four consecutive days. These findings certainly merit further investigation. A study of the identity and life-history of leaf miners on tomato seedlings, by E. R. Speyer and W. J. Parr, shows that the fly responsible is Liriomyza solani. A 5 per cent benzene hexachloride dust was more effective than 5 per cent DDT in destroying adult flies, but both substances can injure tomato seedlings. A study of toxicity of analogues of azobenzene against red spider mite revealed no compound more effective than azo. benzene itself (W. H. Read). Hexaethyl tetraphosphate (HETP) and tetraethyl pyrophosphate (TEPP) destroyed adult red spider mites, but not the eggs of this pest. Both the last-mentioned compounds controlled aphids on carnations.

Chemical studies involve, inter alia, fundamental investigations into the mechanism of nitrification in glasshouse soils by $O$. Owen and G. W. Winsor. Experiments with added carbon show that the lower carbon/nitrogen ratio produces the higher level of nitrification. The availability of alginates raises the interesting possibility of using such substances as sources of organic carbon in soil (O. Owen). Alginic acid and sodium and calcium alginates have given promising results, though ammonium alginate may be almost toxic. Some rather variable results of trials with calcium alginate on a commercial scale, however, show that further work on the subject is needed. Changes in nitrification after steam sterilization are greatly in need of investigation, and it is gratifying to note that work on this project has commenced (J. N. Davies and O. Owen).

A mild criticism of the report is its increase in page size from octavo to quarto, which disorganises the files. This petty complaint, however, serves but to emphasize the value of the work in the report, for he who reads it desires to preserve it for future convenient reference.

\section{EFFECT OF CERTAIN YEASTS ON SOME TUMOUR CELLS}

CERTAIN strains of yeast apparently show in (1 vitro a selectively destructive action on the cells of some malignant tumours, but not on those of benign tumours or of normal tissues. Dr. Giocondo Protti, of Busto Arsizio, Italy, has been working in this field since 1930 and has developed special techniques which he has recently summarized in three articles ${ }^{1}$.
Fragments of tissue were selected from frozen sections examined by phase-contrast microscopy to ensure that only actively growing tissue free from necrosis was used. The fragments were immersed in liquid cultures of Saccharomyces cerevisioe var. ellipsoideus and incubated at $30^{\circ} \mathrm{C}$. for $48 \mathrm{hr}$. About a hundred varieties of yeast were tested, with variable results; certain varieties caused lysis of the nuclei of some tumours but were inactive for other tumours.

Controls with inactivated yeast and with medium devoid of yeast showed that incubation alone or in the presence of certain yeast metabolites did not produce comparable lysis of tumour cells.

Comparative studies on tissues incubated in contact with yeast cultures and afterwards stained with hæmatoxylin and eosin, or better, by the Feulgen tech. nique, showed varying degrees of nuclear lysis in the case of tumour tissues. These degenerative changes could be seen also by phase-contrast microscopy. 'In adenocarcinoma of the alimentary canal, the nuclei of the malignant epithelium showed lysis, whereas the nuclei of the connective tissue and endothelial cells of blood vessels were relatively well preserved. Protti considers the Feulgen reaction a reliable indication, though not amounting to a quantitative measure, of the thymonucleic acid content of nuclei. The nature of the lytic action has not been determined.

In experiments with a spontaneous mammary tumour of mice of $L$-strain, 96 tissue cultures were tested against six different strains of yeast, as com. pared with control tissue cultures of fibroblasts (? mouse), and of retinal epithelium of 12-day chick embryos. Tumour cultures in contact with Saccharomyces cerevisice var. ellipsoideus $E .14$ showed little difference from control cultures and developed normally. By contrast, tumour cultures from the same material in contact with the following strains of Saccharomyces, $E .4, C .35, T .54, E$.VIII and $D$. XII, all showed arrested development and lysis of nuclei in the explants, apart from a few attempts at proliferation modified by the lytic process. Neither fibroblast nor retinal epithelium cultures showed lysis.

The mechanical effect of the yeast particles was controlled by the addition of inert powders, for example, talo or carborundum, in place of yeast cultures. Such additions had no effect on the growth of either tumour or normal tissues in culture. Altogether, 637 experiments gave similar results. Three strains of yeast, $E .4, E .14$ and $E$.VIII, showed no biochemical differences ; but $E .14$ was inactive, while the other two caused lysis of tumour explant.

The lytic effects observed were quite different in appearance from necrobiotic effects on the tumour as shown by the Feulgen technique. Protti considers that the selective lytic action of certain yeasts on certain tumour cells indicates a metabolic difference between tumour and normal cells. In the discussion of his work he expresses the hope that his observations may eventually lead to some form of specific treatment.

It might be objected that normal fibroblasts und chick embryo retinal epithelium may well have different metabolic reactions from mammary tumour or adenocarcinoma of the alimentary canal. Possibly tissue cultures of adolescent mouse mammary tissues and of the benign adenoma that frequently precedes adenocarcinoma of breast in mice would provide better control when studying mouse mammary carcinoma.

P. R. P.

1 Protti, G., Tumori, 22, 222 (1948); 88, 156 (1949); 24, 14 (1956). 